\section{MS46 O1}

Visualizing the dynamics of grains and dislocations structures Henning F. Poulsen ${ }^{\mathrm{a}}$, Bo Jakobsen ${ }^{\mathrm{a}}$, Wolfgang Pantleon $^{\mathrm{a}}$, Erik M. Lauridsen ${ }^{\mathrm{a}}$, Søren Schmidt ${ }^{\mathrm{a}}$, Lawrence Margulies $^{\text {ab }}$, Henning O. Søren-sen ${ }^{\mathrm{a}}$, Dorte Juul Jensen ${ }^{\mathrm{a}}$, Gavin Vaughan ${ }^{\mathrm{b}}$,Ulrich Lienert ${ }^{\mathrm{c}}$, Jonathan Almer $^{\mathrm{c}}$, Sarjvit D. Shastric ${ }^{\mathrm{c}}$, Risø Nat. Lab., Roskilde, Denmark, ${ }^{b}$ ESRF, Grenoble, France. ${ }^{c}$ APS, Argonne Nat.Lab., Chigago, US. E-mail: henning.friis.poulsen@risoe.dk

\section{Keywords: materials application of synchrotron radiation, multicrystal X-ray diffractometry, dislocation structure properties}

3D X-Ray Diffraction (3DXRD) microscopy is an emerging tool for fast and non-destructive characterization of the individual grains and sub-grains inside bulk materials [1]. The method is based on diffraction with hard x-rays, enabling 3D studies of millimetre - centimetre thick specimens. Ray tracing with several detectors is applied. The position, morphology, orientation, and elastic strain can be derived for hundreds of diffracting units (grains or sub-grains) simultaneously. Notably, the method is sufficiently fast for in situ characterization of typical processing steps such as annealing or deformation.

In this talk focus is on recent results within two areas:

- By applying tomographic reconstruction principles, 3D grain maps can be derived. New algorithms are presented which extend the application of such methods also to maps of deformed specimens [2]. First results for dynamic studies of recrystallisation [3] and grain growth are summarized.

- "Ultra-high resolution 3DXRD" is a technique which enables 3D reciprocal maps to be derived of individual deeply embedded dislocation structures. With a resolution of $0.0005 \AA^{-1}$, in favorable conditions, individual dislocations can be probed. First results obtained during tensile deformation revealed unexpected intermittent dynamics of the dislocation structures in pure $\mathrm{Cu}[4]$.

[1] H.F. Poulsen. Three-Dimensional X-Ray Diffraction Microscopy (Spinger, Berlin, 2004).

[2] A. Alpers, G.T. Herman, E. Knudsen, H.F. Poulsen, and L. Rodek, in Advances in Discrete Tomography and its Applications (Birkhäuser, Berlin, 2007)

[3] S. Schmidt, S.F. Nielsen, C. Gundlach, L. Margulies, X. Huang, and D. Juul Jensen, Science (2004) 305, 229-232

[4] B. Jakobsen, U. Lienert, J. Almer, H.O. Sørensen, W. Pantleon, and H.F. Poulsen. Science (2006), 312, 889-892

\section{MS46 O2}

The laws of substructure Nonuniformity of Metals in Terms of X-Ray Diffraction Yuriy Perlovich, Margarita Isaenkova, Vladimir Fesenko, Moscow Engineering Physics Institute, Moscow, Russia.

E-mail: perlovich@,hotmail.com

\section{Keywords: substructure, texture, microstrain}

Any metal material, subjected to a deformation treatment, is characterized by a wide spectrum of substructure conditions and parameters of X-ray lines, obtained as reflections from grains with different orientations by texture measurements. When assuming that the intensity I of X-ray line under other equal conditions depends on the position of reflecting grains relative to texture maxima and minima, the physical half-width $\beta$ characterizes distortion of the crystalline lattice and grain fermentation into coherent domains, the peak position $2 \theta$ depends on the residual elastic microstrain, then observed combinations of these diffraction parameters give an idea of inevitable substructure nonuniformity of material. The distinct mutual correlation was found between statistically significant massifs of measured parameters of the same Xray line for the same metal material, always containing grains in multifarious substructure conditions [1,2]. Combinations of these parameters submit to the general laws, controlling the substructure nonuniformity of metal materials. Discovery of these laws became possible due to recent progress in automation of X-ray diffractometric measurements and computer data treatment.

Under any technological treatment all metal materials acquire that or another crystallographic texture, and texture formation is accompanied by arising of the regular substructure nonuniformity. The orientation of grains relative to texture maxima and minima is the most effective criterion for systematization of substructure inhomogeneities. Minima of the physical broadening of Xray lines coincide with texture maxima, whereas maxima of line broadening are localized within texture minima. The $1^{\text {st }}$ law of substructure nonuniformity connects it with the deformation texture: Residual deformation effects, i.e. grain fragmentation and lattice distortion, are minimal in texture maxima and increase up to highest values by passing to texture minima.

The distribution of residual compressive and extensive elastic microstrains in metal products ensures the microstress equilibrium and correlates with symmetry of the deformation scheme. In rolled metal materials this distribution shows a cross-wise pattern, that is alternation of quadrants with predominance of elastic extension or elastic compression, providing an equilibrium of microstresses relative to the symmetry planes in the rolling scheme. The $2^{\text {nd }}$ law of substructure nonuniformmity concerns variation of lattice parameters in textured metal products due to elastic microstrain: For each grain with crystalline lattice, extended along axis $<\mathrm{hkl}>$ by $(+\varepsilon)$, there is its pair with the symmetric orientation, where along axis $<$ hkl $>$ crystalline lattice is compressed by $(-\varepsilon)$, so that accompanying elastic microstresses are equilibrated.

The $3 \mathrm{~d}$ law of substructure nonuniformity bases on the revealed correlation between physical broadening and peak position of X-ray line: By passing from residual tension of the crystalline lattice to its compression, grain fragmentation becomes stronger, i.e. tensile elastic deformation is more uniform than compressing one.

[1] Perlovich Yu., Bunge H.J., Isaenkova M., text\& Microstr., 1997; 29, 241.

[2] Perlovich Yu., Isaenkova M., Fesenko V., Bunge H.J., Mater. Sci. Forum, 2005, 495-497, 77 